\title{
Adult Neurogenesis in the Hippocampus From a Perspective of Discrimination and Generalization: A Hypothesis
}

\author{
A. PISTIKOVA ${ }^{\mathbf{1}^{*}}$, H. BROZKA ${ }^{\mathbf{1}^{*}}$, A. STUCHLIK $^{\mathbf{1}}$ \\ * These authors contributed equally to this work. \\ ${ }^{1}$ Department of Neurophysiology of Memory, Institute of Physiology of the Czech Academy of \\ Sciences, Prague, Czech Republic
}

Received March 1, 2017

Accepted April 18, 2017

\section{Summary}

The function of adult neurogenesis in the dentate gyrus is not yet completely understood, though many competing theories have attempted to explain the function of these newly-generated neurons. Most theories give adult neurogenesis a role in aiding known hippocampal/dentate gyrus functions. Other theories offer a novel role for these new cells based on their unique physiological qualities, such as their low excitability threshold. Many behavioral tests have been used to test these theories, but results have been inconsistent and often contradictory. Substantial variability in tests and protocols may be at least partially responsible for the mixed results. On the other hand, conflicting results arising from the same tests can serve as aids in elucidating the function of adult neurogenesis. Here, we offer a hypothesis that considers the cognitive nature of tasks commonly used to assess the function of adult neurogenesis, and introduce a dichotomy between tasks focused on discrimination vs. generalization. We view these two aspects as opposite ends of the continuous spectrum onto which traditional tests can be mapped. We propose that high neurogenesis favors behavioral discrimination while low adult neurogenesis favors behavioral generalization of a knowledge or rule. Since many tasks require both, the effects of neurogenesis could be cancelled out in many cases. Although speculative, we hope that our view presents an interesting and testable hypothesis of the effect of adult neurogenesis in traditional behavioral tasks. We conclude that new, carefully designed behavioral tests may be necessary to reach a final consensus on the role of adult neurogenesis in behavior.

\section{Key words}

Adult neurogenesis • Function • Discrimination • Generalization • Spatial memory • Pattern Separation • Review

\section{Corresponding authors}

A. Pistikova and A. Stuchlik, Department of Neurophysiology of Memory, Institute of Physiology of the Czech Academy of Sciences, Vídeňská 1083, 14220 Prague, Czech Republic. E-mail: adela.pistikova@fgu.cas.cz or ales.stuchlik@fgu.cas.cz

Since the discovery of adult neurogenesis in the dentate gyrus of the hippocampus in both rats (Altman and Das 1965) and humans (Eriksson et al. 1998), many scientists have attempted to explore the function of these newly-generated cells. Despite extensive research, however, it is not yet clear what the exact function of adult-born neurons in the hippocampus is (Kempermann 2011, Aimone et al. 2014). Here, we first describe the current theories that attribute a role to adult neurogenesis in behavior. Second, we present our hypothesis along with supporting evidence. In short, we propose that both high and low levels of adult neurogenesis are adaptive - high neurogenesis favors discrimination, and low neurogenesis favors generalization. We believe that our view does not contradict current theories, but offers a novel and unifying concept to explain this phenomenon.

Initially, many studies attempted to assess the contribution of adult neurogenesis to established functions of the hippocampus, e.g. orientation in space. Indeed, new neurons become incorporated into the hippocampal network during spatial learning, which 
suggests that new neurons may participate in the formation of a spatial memory (Kee et al. 2007, Trouche et al. 2009, Snyder et al. 2014) or in memory retrieval (Snyder et al. 2005, Deng et al. 2009, Jessberger et al. 2009). Several studies have shown a deficit in spatial learning in adult neurogenesis-deficient animals (Dupret et al. 2008, Zhang et al. 2008, Garthe et al. 2009, Martinez-Canabal et al. 2013). However, many other studies have failed to find an effect of adult neurogenesis in hippocampal-dependent tasks (Saxe et al. 2006, Hernández-Rabaza et al. 2009, Wojtowicz et al. 2008, Groves et al. 2013, Martinez-Canabal et al. 2013).

Other authors have hypothesized that new neurons may be important for pattern separation a function commonly assigned to the dentate gyrus (Piatti et al. 2013). Pattern separation is a process that transforms similar inputs into less-similar, non-overlapping, outputs (Marr 1971, Schmidt et al. 2012). As described elsewhere, the dentate gyrus is a region ideally suited for the function of pattern separation due to high numbers of granule cells, a low excitation threshold, and perfect anatomical location within the hippocampal circuit. Models that include new neurons in pattern separation have assigned them the function of being the recipients of new information (Aimone and Gage 2011). Experimental evidence agrees with the hypothesis that new neurons contribute to pattern separation, as it has been shown that an ablation or reduction of neurogenesis impairs the discriminationdemanding separation of similar inputs (Saxe et al. 2006, Clelland et al. 2009, Sahay et al. 2011, Nakashiba et al. 2012).

However, newer theoretical models that extend previous hypotheses have taken into account the unique properties of newly generated granule cells. These models ascribed them novel functions such as temporal integration and separation (Aimone et al. 2006, Becker and Wojtowicz 2007). During maturation, young granule cells have a lower excitatory threshold for long-term potentiation and increased synaptic plasticity compared to mature granule cells (Schmidt-Hieber et al. 2004, Ge et al. 2007). They are integrated into the existing hippocampal circuitry 3-4 weeks after their birth (Hastings and Gould 1999, Markakis and Gage 1999, Cameron and McKay 2001), and begin to form functional synapses at around the age of 28 days (van Praag et al. 2002). This lower long-term potentiation threshold and increased synaptic plasticity during maturation may be key properties for explaining the function of new neurons. These newer models propose that new neurons may serve in the temporal separation and temporal integration of events and contexts, extending the classical pattern separation theory. Simply put, because new neurons are active during all events that happen in a short period of time, memories of these events are coded as being more similar because the overlap of the actively maturing neuronal population is high. As time proceeds there is an overturn in the population of these maturing neurons, resulting in more distinct codes for these memories (Aimone et al. 2009). Indeed, time is a very reliable 'separator' of highly similar memories, because no two similar memories ever happen at precisely the same time. The trace eye-blink conditioning task, which requires the formation of an association between temporally close (but non-overlapping) stimuli, was shown to be dependent on intact neurogenesis (Shors et al. 2001). So far, however, no behavioral test has been devised to directly test if neurogenesis is required for making temporally-distant memories more distinct. Nevertheless, it has been shown that when exposure to two contexts are separated by more than three weeks, very distinct cell populations in the dentate gyrus are recruited (Rangel et al. 2014).

Another theory regarding the function of adult neurogenesis is related to aiding the forgetting of old information in favor of more recent. This theory has already gained some empirical support. Akers and colleagues showed that without neurogenesis old memories are maintained, whereas increasing neurogenesis by means of exercise increases forgetting (Akers et al. 2014, Epp et al. 2016). This theory contrasts with the hypothesis of catastrophic interference proposed by Wiscott and colleagues (2006), in which neurogenesis is essential for coding new information without the loss of old information. This is because without neurogenesis old information would be quickly rewritten by newer information. Without the addition of new neurons, the dentate gyrus would be unable to assign unique codes to new memories, resulting in high interference between memories. In a computational model, these latter authors showed that only in a network with neurogenesis can old memories be effectively utilized when needed.

Our hypothesis is not in conflict with any of the above-mentioned theories, but offers a new perspective on the function of adult neurogenesis in the dentate gyrus. We propose that both high and low levels of adult neurogenesis could be adaptive to behavior in different situations. For instance, it is known that neurogenesis increases rapidly following environmental enrichment 
(Kempermann et al. 1997). This is not reminiscent of convalescence from pathological state, but rather seems more like an adaptive change, and may be considered as preparation for encountering a highly-changing habitat (LaDage 2015). Lower levels of neurogenesis, on the other hand, may be beneficial in conserved environments. This way, an animal can maintain its responses even in slightly altered environments. Direct manipulation of neurogenesis can predispose an animal to cope with tasks requiring either high discrimination (increase of neurogenesis) or generalization (decreased neurogenesis). As mentioned above, adult neurogenesis has been most often mentioned as aiding ,pattern separation', which, in behavioral tasks, refers to the ability to discriminate between two similar but distinct contexts. On the other hand, over-generalization, such as in post-traumatic stress disorder (PTSD), has been proposed to be a result of decreased neurogenesis (Kheirbek et al. 2012, Besnard and Sahay 2015). In this disorder, a patient is sensitive to certain aspects of the environment that were present during a traumatic experience. Upon re-contacting these aspects, although in a different context, the patient feels anxious. Generalization is "the act or process whereby a learned response is made to a stimulus similar to but not identical with the conditioned stimulus" (MerriamWebster dictionary). Rule learning is a form of generalization that is part of many behavioral protocols used to study adult neurogenesis (Aimone et al. 2009).

Many complex tasks used to assess the contribution of adult neurogenesis require both differentiating between similar objects and situations (discrimination), and the application of the same rule to a different situation (generalization) in different stages of learning. Our hypothesis is that discrimination and generalization are opposite ends on the same continuous scale, and the degree to which these two capabilities are utilized in each task explains the effect that the manipulation of adult neurogenesis has on the performance of the task. Moreover, individual variations of protocol can push learning requirements more towards context discrimination or generalization, as will be demonstrated in the following examples.

There are two classes of discrimination tasks that are commonly used to test the impact of neurogenesis on behavior: one including contextual discrimination tests and the second including spatial discrimination tests. Context discrimination tests require discrimination of the environment where animal performance is measured, either by the level of freezing in the same context or by comparing freezing between the same and a similar context. Results have been consistent and agree that lower neurogenesis means poorer separation between two similar contexts (Sahay et al. 2011, Niibori et al. 2012, Tronel et al. 2012). The second class of tests where a reduction of neurogenesis decreases performance is spatial discrimination tasks. These tasks usually use a radial-arm maze or a T-maze. Similar tasks use a touch screen to discriminate between two presented choices. Regardless of the specifics, both types of spatial discrimination tasks require selecting one of two choices based on a pre-learned rule. The focus of most studies has been on varying the spatial distance between choices; correct discrimination between spatially proximal choices is regarded to be a measure of pattern separation. Apart from discrimination between two positions, making a correct choice in this task requires an intact working memory. Importantly, this task also requires the animal to generalize a rule across trials. In line with the 'pattern separation' function of the dentate gyrus, animals with reduced neurogenesis show impairment when the presented choices are close to each other but not when far apart (Clelland et al. 2009). However, another study showed that this is true only if spatial discrimination includes reversal, otherwise the separation of stimuli a small distance apart is not dependent on adult neurogenesis (Swan et al. 2014). Similarly, Saxe and colleagues (2007) did not find a deficit when discriminating radial arms close together compared to far apart in neurogenesis deficient animals. In fact, in trials with a high conflict between choices they found a consistent improvement of working memory in adult neurogenesis-deficient animals. When looking closely, there are also other studies which show a trend towards better learning in adult neurogenesis-deficient animals where the rule to learn is more complex. In a non-matchto-sample (NMTS) task a trend towards making more correct choices was found for animals with reduced neurogenesis (by focal irradiation) than for control animals (Hernández-Rabaza et al. 2009). In this task, animals had to select the arm opposite the (rewarded) sample arm to receive the reward. The authors attributed the apparent faster learning in adult neurogenesis deficient animals to the presence of slow learners in the control group. However, in a match-to-sample (MTS) variant in a T-maze task (where the goal was to select the same arm as the sample arm), irradiated animals performed the same as controls. Similarly, the switch from the NMTS to MTS task variant was handled equally 
well by both irradiated and control animals. Importantly, irradiated animals showed just a $36 \%$ reduction of adult neurogenesis. Therefore, it is possible that with a higher reduction in adult neurogenesis irradiated animals would be more efficient. It is noteworthy that this is one of the few tasks that have been used to test the function of adult neurogenesis that do not require precision, and irradiated animals were at least unimpaired.

Another common task used to assess the contribution of neurogenesis is the Morris water maze (MWM). This task yields notoriously conflicting results, which is thought to be caused by the high variety in experimental protocols (Garthe and Kempermann 2013). Since this task challenges both generalization and discrimination, different protocols may challenge each function to a different degree. In the beginning, a rule must be learned - there is a hidden platform located at the same location as it was on a previous swim. After the animal grasps this concept, remembering the precise spatial location of the platform is crucial to quickly escaping onto it. The balance between the two components required to solve this task may cancel each other out. Simply put, the performance of neurogenesis altered animals depends on what each particular MWM protocol challenges more - discrimination or generalization. Superior rule formation was illustrated in an experiment by Deng and colleagues (2009). A MWM deficit was only found in probe trials in neurogenesisdeficient animals, which were conducted several hours post training each day. In the first probe trial there was no deficit in neurogenesis-reduced animals. However, neurogenesis deficient animals showed poorer performance in subsequent probe trials. Although this was interpreted as a decrease of function, the gradual failure to search in a target quadrant is also suggestive of learning - namely, learning a rule that the test that follows acquisition training equals a futile search for the (removed) platform. Similarly, neurogenesis-depleted animals showed a lower spatial (precision) strategy to find a hidden platform (Garthe et al. 2009), although latencies to reach the hidden platform were the same for both groups. On the other hand, juvenile mice with reduced neurogenesis appear to have inferior MWM performance precisely due to the absence of rule understanding, as they keep searching the pool walls (Martinez-Canabal et al. 2013). We propose that due to the challenges to both discrimination and generalization, the contribution of neurogenesis is very difficult to analyze in MWM.
Although not common, there are few tasks in which generalization is required more than discrimination (Aimone et al. 2009). For instance, this can be seen in an improvement of function in a radial arm maze during trials that interfered with previous trials (Saxe et al. 2007). In addition to knowledge of the rule 'the arm opposite the sample arm is correct', it is necessary for the animal to choose a previously presented sample arm to make the correct choice.

Another task that requires generalization more than discrimination is the Carousel maze. In this task the animal avoids an invisible sector of a rotating arena. This task is not intuitive to animals, because they have to disregard cues present within the arena while using extra-arena cues to determine the location. Precise knowledge of the position of the sector is not necessary, because for successful avoidance it is sufficient to move in a counter-rotation direction in any safe part of the maze. Our group found repeatedly superior performance in adult neurogenesis deficient rats in reversal in a Carousel maze task (Brozka et al. 2017).

\section{Conclusions}

Adult neurogenesis is a newly discovered phenomenon that has fascinated scientists for the past two decades. However, despite intensive research we still do not know exactly why new neurons are born. Attempts have been made to establish the function of adult neurogenesis in terms of hippocampal function, such as in facilitating spatial learning or helping the dentate gyrus to separate patterns. In addition, new roles for new neurons have been proposed based on their physiological properties, such as temporal separation/integration and forgetting. To date however, very few tasks have been specifically developed to test new adult neurons. This may be in fact quite difficult, for as we propose here, tasks may require capabilities that utilize neurogenesis in different ways. Our hypothesis merges two observations: (1) low neurogenesis leads to overgeneralization, which is often viewed negatively, such as in PTSD, but is also necessary for making rules; (2) high neurogenesis, on the other hand, leads to high discrimination, which has consistently positive connotations in the field of neuroscience. However, it is not always advantageous to accept a slightly different situation as being completely distinct (as many situations life have taught us). Thus, we propose that there are two sides to both generalization and discrimination; both may be beneficial or detrimental 
depending on the situation (Fig. 1). Environmental cues (such as environmental enrichment or long-term stability) could adjust levels of adult neurogenesis to optimal levels. In addition, species have different ecologies, and the habitat that an animal evolved for may need to be considered when studying the function of adult neurogenesis.

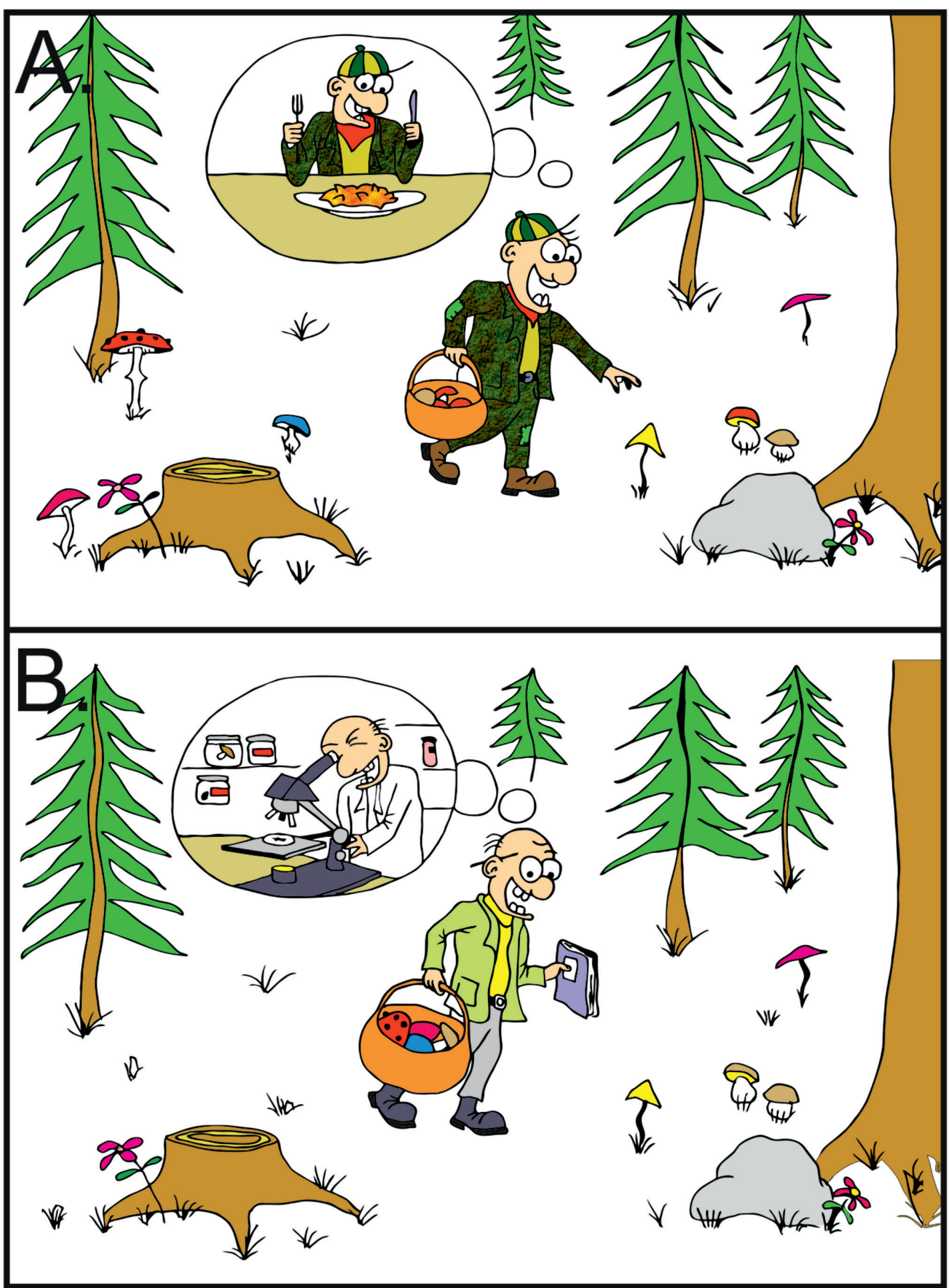

Fig. 1. Generalization and discrimination are beneficial depending on the goal - a difference of situation. If you are a recreational mushroom picker (A) you want to discriminate edible mushrooms from poisonous ones. However, if you are a mycologist (B) you want to collect all types of mushrooms, and it is beneficial to generalize their properties. 


\section{Conflict of Interest}

There is no conflict of interest.

\section{Acknowledgements}

This work was supported by GACR grant 17-04047S. Institutional support was provided by RVO: 67985823 .

\section{References}

AIMONE JB, GAGE FH: Modeling new neuron function: a history of using computational neuroscience to study adult neurogenesis. Eur J Neurosci 33: 1160-1169, 2011.

AIMONE JB, LI Y, LEE SW, CLEMENSON GD, DENG W, GAGE FH: Regulation and function of adult neurogenesis: from genes to cognition. Physiol Rev 94: 991-1026, 2014.

AIMONE JB, WILES J, GAGE FH: Potential role for adult neurogenesis in the encoding of time in new memories. Nat Neurosci 9: 723-727, 2006.

AIMONE JB, WILES J, GAGE FH: Computational influence of adult neurogenesis on memory encoding. Neuron 61: 187-202, 2009.

AKERS KG, MARTINEZ-CANABAL A, RESTIVO L, YIU AP, DE CRISTOFARO A, HSIANG HLL, WHEELER AL, GUSKJOLEN A, NIIBORI Y, SHOJI H, ET AL.: Hippocampal neurogenesis regulates forgetting during adulthood and infancy. Science 344: 598-602, 2014.

ALTMAN J, DAS GD: Autoradiographic and histological evidence of postnatal hippocampal neurogenesis in rats. J Comp Neurol 124: 319-335, 1965.

BECKER S, WOJTOWICZ JM: A model of hippocampal neurogenesis in memory and mood disorders. Trends Cogn Sci 11: 70-76, 2007.

BESNARD A, SAHAY A: Adult hippocampal neurogenesis, fear generalization, and stress. Neuropsychopharmacology 41: 24-44, 2015.

BROZKA H, PISTIKOVA A, RADOSTOVA D, VALES K, SVOBODA J, GRZYB AN, STUCHLIK A: Adult neurogenesis reduction by a cytostatic treatment improves spatial reversal learning in rats. Neurobiol Learn Mem 141: 93-100, 2017

CAMERON HA, MCKAY RD: Adult neurogenesis produces a large pool of new granule cells in the dentate gyrus. J Comp Neurol 435: 406-417, 2001.

CLELLAND CD, CHOI M, ROMBERG C, CLEMENSON GD, FRAGNIERE A, TYERS P, JESSBERGER S, SAKSIDA LM, BARKER RA, GAGE FH, BUSSEY TJ: A functional role for adult hippocampal neurogenesis in spatial pattern separation. Science 325: 210-213, 2009.

DENG W, SAXE MD, GALLINA IS, GAGE FH: Adult-born hippocampal dentate granule cells undergoing maturation modulate learning and memory in the brain. J Neurosci 29: 13532-13542, 2009.

DUPRET D, REVEST JM, KOEHL M, ICHAS F, DE GIORGI F, COSTET P, ABROUS DN, PIAZZA PV: Spatial relational memory requires hippocampal adult neurogenesis. PLoS One 3: e1959, 2008.

ERIKSSON PS, PERFILIEVA E, BJÖRK-ERIKSSON T, ALBORN AM, NORDBORG C, PETERSON DA, GAGE FH: Neurogenesis in the adult human hippocampus. Nat Med 4: 1313-1317, 1998.

GARTHE A, BEHR J, KEMPERMANN G: Adult-generated hippocampal neurons allow the flexible use of spatially precise learning strategies. PLoS One 4: e5464, 2009.

GARTHE A, HUANG Z, KACZMAREK L, FILIPKOWSKI RK, KEMPERMANN G: Not all water mazes are created equal: cyclin D2 knockout mice with constitutively suppressed adult hippocampal neurogenesis do show specific spatial learning deficits. Genes Brain Behav 13: 357-364, 2014.

GARTHE A, KEMPERMANN G.: An old test for new neurons: refining the Morris water maze to study the functional relevance of adult hippocampal neurogenesis. Front Neurosci 7: 63, 2013.

GE S, YANG C, HSU K, MING G, SONG H: A critical period for enhanced synaptic plasticity in newly generated neurons of the adult brain. Neuron 54: 559-566. 2007.

GROVES JO, LESLIE I, HUANG GJ, MCHUGH SB, TAYLOR A, MOTT R, MUNAFÒ M, BANNERMAN DM, FLINT J: Ablating adult neurogenesis in the rat has no effect on spatial processing: evidence from a novel pharmacogenetic model. PLoS Genet 9: e1003718, 2013. 
HASTINGS NB, GOULD E: Rapid extension of axons into the CA3 region by adult-generated granule cells. $J$ Comp Neurol 413: 146-215, 1999.

HERNÁNDEZ-RABAZA V, LLORENS-MARTÍN M, VELÁZQUEZ-SÁNCHEZ C, FERRAGUD A, ARCUSA A, GUMUS HG, GÓMEZ-PINEDO U, PÉREZ-VILLALBA A, ROSELLÓ J, TREJO JL, ET AL.: Inhibition of adult hippocampal neurogenesis disrupts contextual learning but spares spatial working memory, long-term conditional rule retention and spatial reversal. Neuroscience 159: 59-68, 2009.

JESSBERGER S, CLARK RE, BROADBENT NJ, CLEMENSON GD, CONSIGLIO A, LIE DC, SQUIRE LR, GAGE FH: Dentate gyrus-specific knockdown of adult neurogenesis impairs spatial and object recognition memory in adult rats. Learn Mem 16: 147-154, 2009.

KEE N, TEIXEIRA CM, WANG AH, FRANKLAND PW: Preferential incorporation of adult-generated granule cells into spatial memory networks in the dentate gyrus. Nat Neurosci 10: 355-362, 2007.

KEMPERMANN G, KUHN HG, GAGE FH: More hippocampal neurons in adult mice living in an enriched environment. Nature 386: 493-495, 1997.

KEMPERMANN G: Adult Neurogenesis 2. Oxford University Press, New York, 2011.

KHEIRBEK MA, KLEMENHAGEN KC, SAHAY A, HEN R: Neurogenesis and generalization: a new approach to stratify and treat anxiety disorders. Nat Neurosci 15: 1613-1620, 2012.

LADAGE LD: Environmental change, the stress response, and neurogenesis. Integr Comp Biol 55: 372-383, 2015.

MARKAKIS EA, GAGE FH: Adult-generated neurons in the dentate gyrus send axonal projections to field CA3 and are surrounded by synaptic vesicles. J Comp Neurol 406: 449-460, 1999.

MARR D: Simple memory: a theory for archicortex. Philos Trans R Soc Lond B Biol Sci 262: 23-81, 1971.

MARTINEZ-CANABAL A, AKERS KG, JOSSELYN S A, FRANKLAND PW: Age-dependent effects of hippocampal neurogenesis suppression on spatial learning. Hippocampus 23: 66-74, 2013.

NAKASHIBA T, CUSHMAN JD, PELKEY KA, RENAUDINEAU S, BUHL DL, MCHUGH TJ, RODRIGUEZ BARRERA V, CHITTAJALLU R, IWAMOTO KS, MCBAIN CJ, ET AL.: Young dentate granule cells mediate pattern separation, whereas old granule cells facilitate pattern completion. Cell 149: 188-201, 2012.

NIIBORI Y, YU TS, EPP JR, AKERS KG, JOSSELYN SA, FRANKLAND PW: Suppression of adult neurogenesis impairs population coding of similar contexts in hippocampal CA3 region. Nat Commun 3: 1253, 2012.

VAN PRAAG H, SCHINDER AF, CHRISTIE BR, TONI N, PALMER TD, GAGE FH: Functional neurogenesis in the adult hippocampus. Nature 415: 1030-1034, 2002.

RANGEL LM, ALEXANDER AS, AIMONE JB, WILES J, GAGE FH, CHIBA AA, QUINN LK: Temporally selective contextual encoding in the dentate gyrus of the hippocampus. Nat Commun 5: 3181, 2014.

SAHAY A, SCOBIE KN, HILL AS, O'CARROLL CM, KHEIRBEK MA, BURGHARDT NS, FENTON AA, DRANOVSKY A, HEN R: Increasing adult hippocampal neurogenesis is sufficient to improve pattern separation. Nature 472: 466-470, 2011.

SAXE MD, BATTAGLIA F, WANG JW, MALLERET G, DAVID DJ, MONCKTON JE, GARCIA ADR, SOFRONIEW MV, KANDEL ER, SANTARELLI L, ET AL.: Ablation of hippocampal neurogenesis impairs contextual fear conditioning and synaptic plasticity in the dentate gyrus. Proc Natl Acad Sci USA 103: 17501-17506. 2006.

SAXE MD, MALLERET G, VRONSKAYA S, MENDEZ I, GARCIA AD, SOFRONIEW MV, KANDEL ER, HEN R: Paradoxical influence of hippocampal neurogenesis on working memory. Proc Natl Acad Sci USA 104: 4642-4646, 2007.

SHORS TJ, MIESEGAES G, BEYLIN A, ZHAO M, RYDEL T, GOULD E: Neurogenesis in the adult is involved in the formation of trace memories. Nature 410: 372-376, 2001.

SHORS TJ, TOWNSEND DA, ZHAO M, KOZOROVITSKIY Y, GOULD E: Neurogenesis may relate to some but not all types of hippocampal-dependent learning. Hippocampus 12: 578-584, 2002.

SCHMIDT B, MARRONE DF, MARKUS EJ: Disambiguating the similar: the dentate gyrus and pattern separation. Behav Brain Res 226: 56-65, 2012.

SCHMIDT-HIEBER C, JONAS P, BISCHOFBERGER J: Enhanced synaptic plasticity in newly generated granule cells of the adult hippocampus. Nature 429: 3682-3685, 2004. 
SNYDER JS, HONG NS, MCDONALD RJ, WOJTOWICZ JM: A role for adult neurogenesis in spatial long-term memory. Neuroscience 130: 843-852, 2005.

SNYDER JS, KEE N, WOJTOWICZ JM, KANNANGARA TS, EADIE BD, BOSTROM CA, MORCH K, PATRICIA S, CHRISTIE BR, DREW LJ, ET AL.: Effects of adult neurogenesis on synaptic plasticity in the rat dentate gyrus. J Neurophysiol 6: 2423-2431, 2001.

SWAN AA, CLUTTON JE, CHARY PK, COOK SG, LIU GG, DREW MR: Characterization of the role of adult neurogenesis in touch-screen discrimination learning. Hippocampus 24: 1581-1591, 2014.

TRONEL S, BELNOUE L, GROSJEAN N, REVEST JM, PIAZZA PV, KOEHL M, ABROUS DN: Adult-born neurons are necessary for extended contextual discrimination. Hippocampus 22: 292-298, 2012.

TROUCHE S, BONTEMPI B, ROULLET P, RAMPON C: Recruitment of adult-generated neurons into functional hippocampal networks contributes to updating and strengthening of spatial memory. Proc Natl Acad Sci US A 106: 5919-5924, 2009.

WISKOTT L, RASCH MJ, KEMPERMANN G: A functional hypothesis for adult hippocampal neurogenesis: avoidance of catastrophic interference in the dentate gyrus. Hippocampus 16: 329-343, 2006.

WOJTOWICZ JM, ASKEW ML, WINOCUR G: The effects of running and of inhibiting adult neurogenesis on learning and memory in rats. Eur J Neurosci 27: 1494-1502, 2008.

ZHANG CL, ZOU Y, HE W, GAGE FH, EVANS RM: A role for adult TLX-positive neural stem cells in learning and behaviour. Nature 451: 1004-1007, 2008. 